\title{
139. On the Cone of Curves of Algebraic Varieties
}

\author{
By Yujiro Kawamata \\ Department of Mathematics, University of Tokyo \\ (Communicated by Kunihiko Kodarra, M. J. A., Dec. 12, 1983)
}

In this paper we announce a structure theorem on the cone of curves of algebraic varieties defined over a field of characteristic zero. Details will appear elsewhere. This theorem should be one of the key steps toward the theory of minimal models of algebraic varieties. We already have the so-called contraction theorem (Theorem 4), which is a generalization of Castelnuovo's criterion of exceptional curves of the first kind. Our weak cone theorem guarantees the existence of a good extremal ray to be contracted if the model is not minimal. The remaining thing to be proved would be the theorem on elementary transformations (see Reid [5], [6], Kawamata [2]).

1. We fix our notation. Let $X$ be a normal projective variety. We define : $N_{1}(X)=\{1$-cycles on $X\} / \approx \otimes R, N_{Q}^{1}(X)=\{$ line bundles on $X\} /$ $\approx \otimes \boldsymbol{Q}, N^{1}(X)=N_{Q}^{1}(X) \otimes R$, and $\overline{N E}(X)=$ the closed convex cone in $N_{1}(X)$ generated by effective 1 -cycles, where $\approx$ denotes numerical equivalence. $\quad N_{1}(X)$ and $N^{1}(X)$ are dual to each other by intersection pairing. An element $D \in N^{1}(X)$ is called "nef" (numerically effective or numerically semipositive) if $D \geqq 0$ on $\overline{N E}(X)$. Moreover, if $\left(D^{n}\right)>0$ with $n=\operatorname{dim} X$, then $D$ is called "big". We denote by $\operatorname{Div}(X)$ the group of Weil divisors on $X$ and $K_{X} \in \operatorname{Div}(X)$ the canonical divisor on $X$. A "Q-divisor" is an element $D \in \operatorname{Div}(X) \otimes Q . \quad D$ is called "Q-Cartier" if there is a positive integer $a$ such that $a D$ is a Cartier divisor. If $K_{X}$ is $\boldsymbol{Q}$-Cartier, $X$ is called " $\boldsymbol{Q}$-Gorenstein". For $\boldsymbol{Q}$-Cartier divisors we can define intersection numbers with 1-cycles by linearity and thus their numerical classes in $N^{1}(X)$. For $r \in R$ we define $\ulcorner r\urcorner=\min \{t \in Z$; $t \geqq r\}$ and $\{r\}=r+\ulcorner-r\urcorner$. Let $D=\sum a_{i} D_{i}$ be a $\boldsymbol{Q}$-divisor, where $a_{i} \in \boldsymbol{Q}$ and the $D_{i}$ are mutually distinct prime divisors. We define $\ulcorner D\urcorner$ $=\sum\left\ulcorner a_{i}\right\urcorner D_{i}$ and $\{D\}=\sum\left\{a_{i}\right\} D_{i} . \quad X$ is said to have only "canonical singularities" if $X$ is $\boldsymbol{Q}$-Gorenstein and if the following condition is satisfied: there is a resolution of singularities $f: Y \rightarrow X$ such that $a K_{Y}$ $=f^{*}\left(a K_{X}\right)+a \sum a_{i} E_{i}$ with $a_{i} \geqq 0$, where $a$ is a positive integer such that $a K_{X}$ is a Cartier divisor and the $E_{i}$ are exceptional divisors.

Theorem 1 (Kawamata [1] or Viehweg [8]). Let $X$ be a nonsingular projective variety and $D \in \operatorname{Div}(X) \otimes Q$. Assume the following conditions :

(i) $D$ is nef and big and 
(ii) $\{D\}$ has a support with only normal crossings. Then we have $H^{i}\left(X, \mathcal{O}_{X}\left(\ulcorner D\urcorner+K_{X}\right)\right)=0$ for $i>0$.

Let $\pi: X \rightarrow Z$ be a morphism of normal projective varieties. We define: $N_{1}(X / Z)=$ the subspace of $N_{1}(X)$ generated by curves on $X$ which are mapped to points on $Z$ by $\pi, N^{1}(X / Z)=N^{1}(X) / N_{1}(X / Z)^{\perp}$, $N_{Q}^{1}(X / Z)=$ the image of $N_{Q}^{1}(X)$ in $N^{1}(X / Z), \overline{N E}(X / Z)=$ the closed convex cone in $N_{1}(X / Z)$ generated by curves on $X$ which are mapped to points on $Z$ by $\pi$, and $\overline{N E}(X / Z)=\{z \in \overline{N E}(X / Z) ;(D \cdot z) \geqq 0\}$ for $D \in N^{1}(X / Z)$. $N_{1}(X / Z)$ and $N^{1}(X / Z)$ are dual spaces. $D \in N^{1}(X / Z)$ is called "relatively nef" for $\pi$ if $D \geqq 0$ on $\overline{N E}(X / Z)$. Kleiman's criterion [3] says that $D \in N_{Q}^{1}(X / Z)$ is relatively ample if and only if $D>0$ on $\overline{N E}(X / Z)-\{0\}$. We assume that $X$ has only canonical singularities. A "good relative supporting function" $L$ is an element of $N_{Q}^{1}(X)$ which is relatively nef for $\pi$ and such that $F=F_{L}={ }_{\text {def }} L^{\perp} \cap \overline{N E}(X / Z)-\{0\}$ is non-empty and entirely contained in the half space $\left\{z \in N_{1}(X / Z) ;\left(K_{X} \cdot z\right)<0\right\}$. This $F$ is called a "good relative face" supported by $L$. By Kleiman's criterion, it is easy to see that there is a positive integer $a$ such that $a L-K_{X}$ is relatively ample for $\pi$. A good relative face of dimension 1 is called a "good relative extremal ray". In the absolute case, i.e., if $Z$ is a point, $L$ and $F$ are called a "good supporting function" and a "good face" (or a "good extremal ray") of $\overline{N E}(X)$, respectively.

2. Now we state our main theorem. This was first proved by Reid [6] in the case in which $\operatorname{dim} X=3$ and $Z$ is a point. This theorem is "weak", since we do not assert that the $R_{i}$ are locally finite.

Theorem 2. Let $X$ be a projective variety with only canonical singularities, $Z$ a projective variety and let $\pi: X \rightarrow Z$ be a morphism. Then $\overline{N E}(X / Z)$ is a closed convex cone generated by $\overrightarrow{N E}_{K X}(X / Z)$ and all of its good relative extremal rays:

$$
\overline{N E}(X / Z)=\left(\overline{N E}_{K_{X}}(X / Z)+\sum R_{i}\right)^{-},
$$

where the $R_{i}$ are good relative extremal rays and-denotes the closure with respect to the real topology. In particular, if $K_{X}$ is not relatively nef for $\pi$, then $X$ has at least one good relative extremal ray.

The proof needs following theorems which are deduced by using the same method as in Kawamata [2] or Reid [6]. Theorem 3 is a generalization of Theorem 0.2 of Shokurov [7].

Theorem 3. Let $X$ be a non-singular projective variety, $D_{1}, D_{2}$ and $D_{3} \in \operatorname{Div}(X), A \in \operatorname{Div}(X) \otimes \boldsymbol{Q}, r \in \boldsymbol{R}$ with $r>0$ and $r \notin \boldsymbol{Q}$, and $p_{0}$ and $q_{0}$ positive integers with $q_{0}-p_{0} r>0$, which satisfy the following conditions:

(i) $D_{3}$ is nef,

(ii) for arbitrary positive integers $p$ and $q$ such that $q-p r \leqq q_{0}$ $-p_{0} r$ and $q \geqq q_{0}$, there exists a positive integer such that $p D_{1}+q D_{2}$ 
$+s D_{3}+A-K_{X}$ is nef and big, and

(iii) $\ulcorner A\urcorner$ is effective and $\{A\}$ has a support with only normal crossings.

Then there exists some $\varepsilon \in \boldsymbol{R}$ with $0<\varepsilon<q_{0}-p_{0} r$ such that

$$
H^{0}\left(X, \mathcal{O}_{X}\left(p D_{1}+q D_{2}+s D_{3}+\ulcorner A\urcorner\right)\right) \neq 0
$$

for all positive integers $p, q$, and $s$ with $q-p r<\varepsilon$ and $0 \ll q \ll s$.

Theorem 4. Let $X$ be a projective variety with only canonical singularities and $H$ a Cartier divisor on $X$. Assume that $H$ is nef and that $a H-K_{X}$ is nef and big for some positive integer a. Then the linear system $|m H|$ is base point free for $m \gg 0$.

Let $F$ be a good face of $\overline{N E}(X)$ with a good supporting function $L$. By applying Theorem 4 to $L$, we obtain a surjective morphism $\pi: X \rightarrow Z$ to a normal projective variety $Z$ with connected fibers such that for an arbitrary curve $C$ on $X, \pi(C)$ is a point on $Z$ if and only if $\mathrm{cl}(C) \in F$. This $\pi$ is called a "contraction morphism associated to $F$ ". Note that $-K_{X}$ is relatively ample for $\pi$.

Theorem 5. Let $X$ be a projective variety with only canonical singularities, $Z$ a projective variety, $\pi: X \rightarrow Z$ a morphism and $H$ an ample Cartier divisor on $X$. Assume that $K_{X}$ is not relatively nef for $\pi$. Set

\section{Then $r \in \boldsymbol{Q}$.}

$$
r=\max \left\{t \in R ; H+t K_{X} \text { is relatively nef for } \pi\right\} \text {. }
$$

Theorem 6. Let $X$ be a projective variety with only canonical singularities, $Z$ a projective variety, $\pi: X \rightarrow Z$ a morphism, and $L$ a good relative supporting function of $\overline{N E}(X / Z)$ with a good relative face $F$. Then there exists an ample divisor $M$ on $Z$ such that $L+\pi^{*} M$ is a good supporting function of $\overline{N E}(X)$ whose good face is the same as $F$. In other words, a good relative face of $\overline{N E}(X / Z)$ is also a good face of $\overline{N E}(X)$.

Corollary 7. Let $X, Z$ and $\pi$ be as in the theorem. Let $F$ be a good face of $\overline{N E}(X)$ contained in $\overline{N E}(X / Z)$ and $\psi: X \rightarrow W$ the contraction morphism associated to $F$. Then we have a morphism $\mu: W \rightarrow Z$ such that $\pi=\mu \circ \psi$. Moreover the following dual sequences

$$
0 \longrightarrow N_{1}(X / W) \longrightarrow N_{1}(X / Z) \longrightarrow N_{1}(W / Z) \longrightarrow 0
$$

and

$$
0 \longleftarrow N^{1}(X / W) \longleftarrow N^{1}(X / Z) \longleftarrow N^{1}(W / Z) \longleftarrow 0
$$

are exact.

This corollary says that $\overline{N E}(X / Z)$ has an "edge" at a good relative face $F$.

Remark. Our proof is also valid in the case where $X$ is a nonprojective complete algebraic variety with only log-terminal singularities. 


\section{References}

[1] Y. Kawamata: A generalization of Kodaira-Ramanujam's vanishing theorem. Math. Ann., 261, 43-46 (1982).

[2] - Elementary contractions of algebraic 3-folds (to appear in Ann. of Math.).

[ 3 ] S. Kleiman: Toward a numerical theory of ampleness. Ann. of Math., 84, 293-344 (1966).

[4] S. Mori: Threefolds whose canonical bundles are not numerically effective. ibid., 116, 133-176 (1982).

[5] M. Reid: Minimal model of canonical 3-folds. Advanced Studies in Pure Math., vol. 1, Kinokuniya and North-Holland, pp. 131-180 (1983).

[6] - Projective morphisms according to Kawamata (Warwick) (preprint).

[7] V. V. Shokurov: Theorem on non-vanishing (preprint).

[8] E. Viehweg: Vanishing theorems. J. reine angew. Math., 335, 1-8 (1982). 